\title{
Geodetic Systems on Linear and Affine Groups. Classics and Quantization.
}

\author{
Jan J. SEAWIANOWSKI \\ Institute of Fundamental Technological Research, Polish Academy of Sciences, \\ Świętokrzyska 21, 00-049 Warsaw, Poland \\ E-mail: jslawian@ippt.gov.pl
}

This article is part of the Proceedings titled "Geometrical Mathods in Physics: Bialowieza XXI and XXII"

\begin{abstract}
Described are classical and quantized systems on linear and affine groups. Unlike the traditional models applied in astrophysics, nuclear physics, molecular vibrations and elasticity, our models are not only kinematically ruled by the affine group, but also their kinetic energies are affinely invariant. There are geodetic $\operatorname{SL}(n, \mathbf{R})$-invariant models with an open family of bounded solutions and with discrete spectra on the quantized level. They seem to be applicable in nuclear physics, theory of defects in solids, astrophysics, dynamics of inclusions, small droplets of fluids and gas bubbles. Independently of these hypothetical applications, they are interesting in themselves.
\end{abstract}

Analytically, the configuration space $Q$ of affinely-rigid body in $n$ dimensions may be identified with the proper affine group $\operatorname{GAf}^{+}(n, \mathbf{R}) \simeq \mathrm{GL}^{+}(n, \mathbf{R}) \times{ }_{s} \mathbf{R}^{n}$ (the semi-direct product is meant here). The configuration $q=(\Phi, x) \in Q$ is to be understood in such a way that the material point with reference (Lagrange) coordinates $a \in \mathbf{R}^{n}$ occupies the spatial position with the current (Euler) coordinates $q(a, t)=\Phi(t) a+x(t) ; x$ denotes the instantaneous position of the center of mass. Lagrange coordinates are chosen in such a way that the center of mass is characterized by $a=0$. If the center of mass motion is frozen or neglected, the configuration space reduces to $Q_{\text {int }}$, identified with $\mathrm{GL}^{+}(n, \mathbf{R})$. If one does not deal with continuous medium but with a finite or countable system of material points, we can also admit some elements of $\mathrm{GL}^{-}(n, \mathbf{R})$, i.e., the coset of $\mathrm{GL}(n, \mathbf{R})$ consisting of orientation-reversing transformations; also some singular elements are admissible. In any case, the configuration space is then an open subset of $\mathrm{L}(n, \mathbf{R})$ containing $\mathrm{GL}^{+}(n, \mathbf{R}), \mathrm{GL}^{-}(n, \mathbf{R})$ and nontrivially intersecting the manifold of matrices with vanishing determinants. If we impose additional constraints of incompressible, rigid or shape-preserving motions, $\mathrm{GL}^{+}(n, \mathbf{R})$ is replaced respectively by $\mathrm{SL}(n, \mathbf{R}), \mathrm{SO}(n, \mathbf{R})$, or $\mathbf{R}^{+} \mathrm{SO}(n, \mathbf{R})=e^{\mathbf{R}} \mathrm{SO}(n, \mathbf{R})$. Lie-algebraic objects, i.e., affine velocities, will be denoted by $\Omega=(d \Phi / d t) \Phi^{-1}$ and $\hat{\Omega}=\Phi^{-1}(d \Phi / d t)=\Phi^{-1} \Omega \Phi$, where $\Omega$ is invariant under right regular translations in $Q_{\text {int }}$ and suffers the adjoint rule under left regular translations, and $\hat{\Omega}$ behaves in an opposite way with the proviso that right translations act through the inverse adjoint rule. Let us stress that the left regular translations describe transformations acting 
in the physical space, whereas the right ones describe transformations of the body material itself. $\Omega$ and $\hat{\Omega}$ represent essentially the same kinematical object respectively in laboratory (space-fixed) and co-moving (body-fixed) representations. Similarly, translational velocity $v=d x / d t$ may be represented in the co-moving terms by $\hat{v}=\Phi^{-1} v$.

When the affine body is subject to the mentioned constraints, the affine velocities become elements of the corresponding Lie subalgebras of $\mathrm{L}(n, \mathbf{R})$, thus, respectively the traceless, skew-symmetric, or skew-symmetric combined with unity-proportional matrices. In other words, one is dealing then with Lie algebras $\mathrm{SL}(n, \mathbf{R})^{\prime}, \mathrm{SO}(n, \mathbf{R})^{\prime}$, or $\mathbf{R} \otimes \mathrm{SO}(n, \mathbf{R})^{\prime}$. It is geometrically interesting to consider rotationless motion, when $\Omega$ is permanently symmetric, i.e., the case complementary to $\mathrm{SO}(n, \mathbf{R})^{\prime}$. As symmetric matrices do not form a Lie algebra, such constraints are non-holonomic and may be studied both on the d'Alembert and sub-Riemannian (VAK-onomic) level. Something similar (but non-equivalent) may be done on the co-moving level of $\hat{\Omega}$. Obviously, in the rigid body case the skew-symmetric $\Omega, \hat{\Omega}$ become the usual angular velocity in laboratory and co-moving representations.

The dual quantities of $\Omega, \hat{\Omega}$, i.e., linear $\mathbf{R}$-valued functions of affine velocities, are identified with elements $\Sigma, \hat{\Sigma}$ of the same space $\mathrm{L}(n, \mathbf{R}) \simeq \mathrm{L}(n, \mathbf{R})^{*}$ in the sense of pairing $<\Sigma, \Omega>=<\hat{\Sigma}, \hat{\Omega}>=\operatorname{Tr}(\Sigma \Omega)=\operatorname{Tr}(\hat{\Sigma} \hat{\Omega})$. The quantities $\Sigma, \hat{\Sigma}$ are respectively Hamiltonian generators of the left and right regular translations on $Q_{\text {int }}$. Poisson brackets for $\Sigma, \Sigma$ are determined by the structure constants for $\mathrm{GL}^{+}(n, \mathbf{R})$, those for $\hat{\Sigma}, \hat{\Sigma}$ have the reversed sign, and the mutual ones between $\Sigma$ and $\hat{\Sigma}$ do vanish. By analogy to rigid body mechanics, $\Sigma$ and $\hat{\Sigma}$ are referred to as the affine spin, or hypermomentum, in laboratory and spatial representations. The mutual relationship between them and their transformation rules are identical with those for $\Omega$ and $\hat{\Omega}$. When the aforementioned group-based constraints are imposed, the dual objects of $\Omega, \hat{\Omega}$ also may be identified (through the trace-pairing) with the same subspaces to which they themselves belong, e.g., for the $\mathrm{SO}(n, \mathbf{R})$-rigid body $\Sigma$ and $\hat{\Sigma}$ are skew-symmetric. For the general affine body $S:=\Sigma-\Sigma^{T}$ and $V:=\hat{\Sigma}-\hat{\Sigma}^{T}$ generate left and right orthogonal translations on $Q_{\text {int }}$ and are referred to respectively as spin and vorticity [3]. To preserve the correspondence with some classical 3-dimensional formulas we shall use the following convention for the pairing between canonical spins and angular velocities: $\langle\Sigma, \Omega>=<\hat{\Sigma}, \hat{\Omega}>=(1 / 2) \operatorname{Tr}(\Sigma \Omega)=(1 / 2) \operatorname{Tr}(\hat{\Sigma} \hat{\Omega})$. The double summation over the repeating terms is then avoided. It is interesting that $V$ is not a co-moving representation of $S$.

The linear (translational) momentum $p \in \mathbf{R}^{n *}$ is a linear functional on translational velocities, acting through the pairing $\langle p, v\rangle=p_{i} v^{i}=p^{T} v=\operatorname{Tr}(p v)$. The total affine momentum with respect to the origin of spatial coordinates $x^{i}$ (total hypermomentum) consists of the translational and internal parts (in analogy to the angular momentum its doubled skew-symmetric part): $I_{b}^{a}=\Lambda_{b}^{a}+\Sigma^{a}{ }_{b}=x^{a} p_{b}+\Sigma_{b}^{a}$. Poisson brackets for $I$ and $\Lambda$ are identical with those for $\Sigma$, the brackets between $\Lambda$ and $\Sigma$ do vanish. The quantities $I$ are Hamiltonian generators of the center-affine mappings preserving the origin of coordinates. Shifting the origin by $\xi \in \mathbf{R}^{n}$, we modify $\Lambda_{b}^{a}$ by $\xi^{a} p_{b}$.

Of particular geometric interest are kinetic energies (geodetic models), i.e., Riemann structures on groups, which are invariant under the group of all left or right or both regular translations. For the macroscopic elastic systems with affine degrees of freedom such models might seem "academic". Nevertheless, they are not only geometrically interesting and related to the dynamics of integrable lattices, but also hopefully seem to be applicable in nuclear dynamics, astrophysics and in certain non-standard problems of continuum 
mechanics, e.g., defect theory. There is also a correspondence between them and the Arnold description of ideal fluids as Hamiltonian systems on the diffeomorphism group [2]. $\operatorname{SDiff}\left(\mathbf{R}^{n}\right)$ is then "discretized" to $\operatorname{SL}(n, \mathbf{R})$.

Let us begin with the translationless case, when only internal (relative) motion is taken into account and configuration space is identified with $Q_{\text {int }}$. The most general "kinetic energy" (metric structure) on $Q_{\text {int }}$ invariant under all left and right regular translations has the form: $T_{\text {int }}^{\mathrm{KC}}=(A / 2) \operatorname{Tr}\left(\Omega^{2}\right)+(B / 2)(\operatorname{Tr} \Omega)^{2}=(A / 2) \operatorname{Tr}\left(\hat{\Omega}^{2}\right)+(B / 2)(\operatorname{Tr} \hat{\Omega})^{2}$. As $\operatorname{SL}(n, \mathbf{R})$ is semisimple and non-compact, it is never positively definite, apparently, non-acceptable as a kinetic energy. Geometrically, $T_{\mathrm{int}}^{\mathrm{KC}}$ is an affine counterpart of a free and materially-invariant gyroscope with two generalized constant inertial scalars $A$ and $B$. Nevertheless, it turns out that just such a model is convenient and may encode partially a large part of elastic interactions without deriving them from a separate potential term. The above expression reduces to the Killing form of $\operatorname{GL}(n, \mathbf{R})$ when $A=2 n$ and $B=-2$. This special case is mechanically useless, because the corresponding metric is degenerate due to non-semisimplicity of $\operatorname{GL}(n, \mathbf{R})$. It is always so when generalized inertial moments satisfy $A / B=-n$. The dominant $A$-term has the signature $(n(n+1) / 2)+, n(n-1) / 2-)$ (respectively non-compact and compact dimensions) and with the normalization $B=-2$ it reduces on $\operatorname{SL}(n, \mathbf{R})$ to its Killing tensor with the signature $((n(n+1) / 2-1)+, n(n-1) / 2-)$. After performing the Legendre transformation we obtain from $T_{\mathrm{int}}^{\mathrm{KC}}$ the following geodetic Hamiltonian: $\Im_{\text {int }}^{\mathrm{KC}}=(1 / 2 A) \operatorname{Tr}\left(\Sigma^{2}\right)-(B / 2 A(A+n B))(\operatorname{Tr} \Sigma)^{2}=(1 / 2 A) \operatorname{Tr}\left(\hat{\Sigma}^{2}\right)-(B / 2 A(A+n B))(\operatorname{Tr} \hat{\Sigma})^{2}$. Without translational motion both $\Sigma$ and $\hat{\Sigma}$ are constants of motion, i.e., they have the vanishing Poisson brackets with the above $\Im_{\text {int }}^{K C}$. Let us observe that without any external potential the above purely geodetic models are not useful for describing deformative motion, although not so strongly as those based on the d'Alembert principle [4]. The catastrophic phenomena happen in the purely dilatational sector. Namely, volume of the body suffers an exponential expansion or contraction (its logarithm moves uniformly). So, the body may collapse or expand in a non-limited way, although both catastrophic phenomena hold in an infinite time (in the usual d'Alembert model [6] the contraction and return to the normal size in geodetic motion are possible after a finite lapse of time). There exist constant-volume solutions, but they are exponentially unstable. Therefore, this part of motion must be stabilized by external potentials [5], e.g., of the form: $V_{\text {dil }}=k\left(l^{2}+l^{-2}-2\right) / 8=k(\operatorname{ch}(2 q)-1) / 4$ or $V_{\text {dil }}=k\left(\operatorname{th}^{2} q-1\right) / 2$, where $k>0, l=\exp (q)$, $\operatorname{det} \Phi=l^{n}, \Phi=l \phi, \phi \in \mathrm{SL}(n, \mathbf{R})$. These phenomenological models predict anharmonic volume vibrations about the equilibrium $\operatorname{det} \Phi=1$, i.e., $l=1, q=0$. The second of them admits the collapse and unlimited expansion when some energy threshold is exceeded. One can also quite easily construct phenomenological models admitting dissociation but preventing contraction. When it is not $\operatorname{det} \Phi$ or $l$ but just $q$ itself that is used as a primary variable, one can simply postulate the harmonic model: $V_{\text {dil }}=k q^{2} / 2, k>0$ (but there is no physical symmetry between the collapse and expansion).

It is clear that the general solution of geodetic Killing-Casimir models $\left(T_{\mathrm{int}}^{\mathrm{KC}}\right.$ and $\left.\Im_{\text {int }}^{\mathrm{KC}}\right)$ is given by one-parameter subgroups of $\mathrm{GL}^{+}(n, \mathbf{R})$ and their cosets, i.e., $\Phi(t)=\exp (E t) \Phi_{0}=$ $\Phi_{0} \exp (\hat{E} t)$, where $\Phi_{0} \in \mathrm{GL}^{+}(n, \mathbf{R})$ and $E \in \mathrm{GL}(n, \mathbf{R})^{\prime} \simeq \mathrm{L}(n, \mathbf{R})$ are arbitrary; obviously, $\hat{E}=\Phi_{0}^{-1} E \Phi_{0}$ also runs over the whole of $\mathrm{L}(n, \mathbf{R})$. The pairs $\left(\Phi_{0}, E\right)$ or $\left(\Phi_{0}, \hat{E}\right)$ are differently expressed initial conditions, namely $\Phi_{0}=\Phi(0)$ is an initial configuration and $E=\Omega(0), \hat{E}=\hat{\Omega}(0)$ are initial constant values of the affine velocity. The initial value of 
generalized velocity is $\dot{\Phi}(0)=E \Phi_{0}=\Phi_{0} \hat{E}$. The same holds for an incompressible body, when $Q_{\text {int }}=\operatorname{SL}(n, \mathbf{R})$. Obviously, then $\Phi_{0} \in \mathrm{SL}(n, \mathbf{R}) ; E, \hat{E} \in \operatorname{SL}(n, \mathbf{R})^{\prime}$ (traceless) and there is no $B$-term in $T_{\text {int }}^{\mathrm{KC}}$ and $\Im_{\text {int }}^{\mathrm{KC}}$.

As mentioned, the size dynamics of geodetic models on $\mathrm{GL}^{+}(n, \mathbf{R})$ is non-stable. To help against this in a minimal-correction way, one has to introduce a stabilizing dilatational potential $V_{\text {dil }}(\operatorname{det} \Phi)$, like above. First, let us introduce some auxiliary symbols $\omega:=$ $(d \phi / d t) \phi^{-1}$ and $\hat{\omega}:=\phi^{-1} d \phi / d t ; \omega, \hat{\omega} \in \mathrm{SL}(n, \mathbf{R})^{\prime}$. Obviously, $\Omega=\omega+(d q / d t) \operatorname{Id}, \hat{\Omega}=$ $\hat{\omega}+(d q / d t)$ Id (as usual, Id denotes the identity matrix). Similarly, for the affine spin we have the splitting: $\Sigma=\sigma+(p / n) \operatorname{Id}, \hat{\Sigma}=\hat{\sigma}+(p / n) \operatorname{Id}, \sigma, \hat{\sigma} \in \operatorname{SL}(n, \mathbf{R})^{\prime}$, where $p$ denotes dilatational canonical momentum, and $\operatorname{Tr}(\Sigma \Omega)=\operatorname{Tr}(\hat{\Sigma} \hat{\Omega})=\operatorname{Tr}(\sigma \omega)+p \dot{q}=\operatorname{Tr}(\hat{\sigma} \hat{\omega})+p \dot{q}$. Poisson brackets for $\sigma$ are determined by the structure constants of $\operatorname{SL}(n, \mathbf{R})$, those for $\hat{\sigma}$ have reversed signs, and obviously, the mixed ones do vanish. Similarly, all Poisson brackets between shape (shear) and dilatational quantities are vanishing.

Kinetic energies $T_{\text {int }}^{\mathrm{KC}}$ and $\Im_{\mathrm{int}}^{\mathrm{KC}}$ split additively in the shear-dilatation sense,

$$
T_{\mathrm{int}}^{\mathrm{KC}}=\frac{A \operatorname{Tr}\left(\omega^{2}\right)}{2}+\frac{n(A+n B) \dot{q}^{2}}{2}=T_{\mathrm{sh}}+T_{\mathrm{dil}}, \Im_{\mathrm{int}}^{\mathrm{KC}}=\frac{\operatorname{Tr}\left(\sigma^{2}\right)}{2 A}+\frac{p^{2}}{2 n(A+n B)}=\Im_{\mathrm{sh}}+\Im_{\mathrm{dil}} .
$$

These expressions are related to each other via Legendre transformation: $\sigma=A \omega, p=$ $n(A+n B) \dot{q}$. Obviously, $(\omega, \sigma)$ may be replaced in these formulas by $(\hat{\omega}, \hat{\sigma})$. When dilatations are controlled, we use Lagrangians and Hamiltonians of the form: $L=L_{\mathrm{sh}}+L_{\mathrm{dil}}=$ $T_{\text {sh }}+T_{\text {dil }}+V(q), H=H_{\text {sh }}+H_{\text {dil }}=\Im_{\text {sh }}+\Im_{\text {dil }}+V(q)$. Dilatational and shear motions are then completely independent, and they remain so if we admit in addition a purely shear potential $V_{\mathrm{sh}}(\phi)$. The question arises however whether there exists a sufficiently large family of bounded, vibrating solutions when $V_{\mathrm{sh}}=0$, i.e., when the isochoric motion is free (geodetic). It is obvious that

Proposition 1. If $\alpha \in \mathrm{SL}(n, \mathbf{R})^{\prime}$ is similar to a skew-symmetric matrix $\lambda \in \operatorname{SO}(n, \mathbf{R})^{\prime}$ (thus $\lambda=-\lambda^{T}$ ), i.e., if there exists $\Psi \in \mathrm{SL}(n, \mathbf{R})$ such that $\alpha=\Psi \lambda \Psi^{-1}$, then every motion $\phi(t)=\exp (\alpha t) \phi_{0}$ is bounded. It is clear that for $n=2,3$ such motions are periodic (for $n>3$ it may be so but need not).

The question is of course "how large" this subset of the general solution is. It turns out that it has the dimension of $\operatorname{SL}(n, \mathbf{R})$, i.e., $\left(n^{2}-1\right)$.

Proposition 2. The subset of $\operatorname{SL}(n, \mathbf{R})^{\prime}$ consisting of matrices $\Psi \lambda \Psi^{-1}$, where $\lambda$, $\Psi$ as in Proposition 1, contains an open neighborhood of the null matrix $\mathcal{O}$ in $\operatorname{SL}(n, \mathbf{R})^{\prime}$.

Roughly speaking, the set of such matrices is controlled by $\left(n^{2}-1\right)$ parameters. Deforming skew-symmetric matrices by means of similarity transformations we can infinitesimally move in any direction in $\operatorname{SL}(n, \mathbf{R})^{\prime}$. This is seen from the polar decomposition $\Psi=U L$, where $U \in \mathrm{SO}(n, \mathbf{R}), L^{T}=L$, $\operatorname{det} L=1$. Deforming $\mathrm{SO}(n, \mathbf{R})^{\prime}$ with the help of $U$ similarities we obtain just $\mathrm{SO}(n, \mathbf{R})^{\prime}$ itself, i.e., the subspace of skew-symmetric matrices. The point is what we obtain from deformations $\mathrm{SO}(n, \mathbf{R})^{\prime} \ni \lambda \mapsto L \lambda L^{-1}$ using the symmetric $L$-matrices. In terms of infinitesimal arguments this may be transformed into the question concerning the structure of the commutator set $\left\{[\alpha, \lambda]: \alpha^{T}=\alpha, \operatorname{Tr}(\alpha)=0, \lambda^{T}=-\lambda\right\}$. But it is clear from the structure of commutation relations of $\operatorname{GL}(n, \mathbf{R})^{\prime} \simeq \mathrm{L}(n, \mathbf{R})$ (antisymmetric-antisymmetric, antisymmetric-symmetric, symmetric-symmetric) that the 
above commutator set just consists of all symmetric trace-less matrices. Properties of the matrix exponent imply that

Corollary 1. General solutions $\phi(t)=\exp (\alpha t) \phi_{0}=\phi_{0} \exp (\hat{\alpha} t)$ of the $\operatorname{SL}(n, \mathbf{R})$-restricted geodetic problem $\left(T_{\mathrm{int}}^{\mathrm{KC}}, \Im_{\mathrm{int}}^{\mathrm{KC}}\right)$ contains an open subset of bounded solutions, where $\alpha$, $\hat{\alpha}=\phi_{0}^{-1} \alpha \phi_{0} \in \mathrm{SL}(n, \mathbf{R})^{\prime}$, and "open" is meant in the sense of these initial conditions. For any stabilizing dilatational potential $V(q)$ there exists an open subset of general solutions for $L$ and $H$ consisting of bounded motions of the form: $\Phi(t)=\exp (\alpha t+q(t)) \phi_{0}=$ $\phi_{0} \exp (\hat{\alpha} t+q(t))$, where $t \mapsto q(t)$ is a general solution of the one-dimensional Hamiltonian problem with the Lagrangian $L_{\text {dil }}=T_{\text {dil }}-V(q)$ and Hamiltonian $H_{\text {dil }}=\Im_{\text {dil }}+V(q)$.

In this sense some kind of shear dynamics (shape vibrations) may be encoded in a purely geodetic left and right invariant model on $\mathrm{SL}(n, \mathbf{R})$.

Let us now return to $\operatorname{GAf}(n, \mathbf{R}) \simeq \operatorname{GL}(n, \mathbf{R}) \times{ }_{s} \mathbf{R}^{n}$, i.e., include the translational motion. There is no symmetry under right $\mathbf{R}^{n}$-translations, because Lagrange coordinates $a=0$ of the center of mass are fixed and realistic bodies have a finite size. But the full group of left regular translations in $\operatorname{GAf}(n, \mathbf{R})$ (including the spatial $\mathbf{R}^{n}$-translations) is physically meaningful. The total kinetic energy is postulated as a sum $T=T_{\operatorname{tr}}+T_{\text {int }}$ of translational and internal parts. There are no models simultaneously invariant under the left and right action of $\operatorname{GL}(n, \mathbf{R})$. This is due to the very specific non-semisimplicity of $\operatorname{GAf}(n, \mathbf{R})$. Translational kinetic energy invariant under the left action of $\operatorname{GL}(n, \mathbf{R})$ is given by: $T_{\mathrm{tr}}=(m / 2) \hat{v}^{T} \hat{v}=(m / 2) v^{T} C v$, where $C=\phi^{-1 T} \phi^{-1}$ is the Cauchy deformation tensor. Now $T_{\mathrm{tr}}$ is not any longer invariant under the right regular action of $\operatorname{GL}(n, \mathbf{R})$, but only under the right regular action of $\mathrm{O}(n, \mathbf{R})$. In the geodetic case (more generally for $x$ independent potentials) the canonical linear momentum $p_{i}=C_{i j} v^{j}$ is a constant of motion, but the velocity $v^{i}$ is not so (the "drunk-missile" effect). Geodetics in $\mathrm{GL}^{+}(n, \mathbf{R}) \times{ }_{s} \mathbf{R}^{n}$ do not project onto geodetics (straight lines) in $\mathbf{R}^{n}$. For the geodetic model the total affine momentum $I_{b}^{a}$ is a constant of motion, but the affine spin $\Sigma^{a}{ }_{b}$ itself is not so. Translational kinetic energies invariant under the right action of $\mathrm{GL}(n, \mathbf{R})$ have the classical form: $T_{\text {int }}=$ $(m / 2) v^{T} v=(m / 2) \hat{v}^{T} G \hat{v}$, where $G=\phi^{T} \phi$ is the Green deformation tensor. What concerns the left regular action, there is only the orthogonal $\mathrm{O}(n, \mathbf{R})$-invariance, but no higher one.

These invariance properties (shared by those of the total $T$ ) fix our attention on the subclass of kinetic energies (metrics) on $Q_{\text {int }}=\operatorname{GL}^{+}(n, \mathbf{R})$ which are invariant under the right action of $\mathrm{GL}^{+}(n, \mathbf{R})$ and left action of $\mathrm{SO}(n, \mathbf{R}): T_{\text {int }}^{\text {right }}=(J / 2) \operatorname{Tr}\left(\Omega^{T} \Omega\right)+$ $(A / 2) \operatorname{Tr}\left(\Omega^{2}\right)+(B / 2)(\operatorname{Tr} \Omega)^{2}$, or left invariant under $\mathrm{GL}^{+}(n, \mathbf{R})$ and right invariant under $\mathrm{SO}(n, \mathbf{R}): T_{\text {int }}^{\text {left }}=(J / 2) \operatorname{Tr}\left(\hat{\Omega}^{T} \hat{\Omega}\right)+(A / 2) \operatorname{Tr}\left(\hat{\Omega}^{2}\right)+(B / 2)(\operatorname{Tr} \hat{\Omega})^{2}$. In both formulas $J, A$, and $B$ are constant generalized scalar moments of inertia. Obviously, the second and third terms in both formulas are respectively equal to each other. There is an open range of $(J, A, B) \in \mathbf{R}^{n}$ for which $T_{\text {int }}^{\text {right }}$ and $T_{\text {int }}^{\text {left }}$ are positively definite.

It is important that now the general solution of the corresponding geodetic problem is not any longer given by one-parameter subgroups and their cosets $\Phi(t)=\exp (E t) \Phi_{0}=$ $\Phi_{0} \exp (\hat{E} t)$. Nevertheless, such solutions do exist and form a continuous family.

Proposition 3. For geodetic models $T_{\mathrm{int}}^{\mathrm{right}}$ there exist solutions $\Phi^{\mathrm{right}}(t)=\exp (E t) \Phi_{0}$, where $\Phi_{0}$ is arbitrary, and $E$ is normal, i.e., $\left[E^{T}, E\right]=0$ (e.g., symmetric or skewsymmetric). Similarly, for $T_{\mathrm{int}}^{\text {left }}$ there exist solutions $\Phi^{\text {left }}(t)=\Phi_{0} \exp (E t)$. The same holds for incompressible models, i.e., there are respectively solutions of the form $\phi^{\text {right }}(t)=$ $\exp (\alpha t) \phi_{0}, \phi^{\text {left }}(t)=\phi_{0} \exp (\alpha t)$, where $\phi_{0}$ is arbitrary, and $\alpha$ satisfies $\left[\alpha^{T}, \alpha\right]=0$. 
These are affine counterparts of stationary rotations (relative equilibria [1, 2]) in rigid body mechanics. For the compressible motion this result may be easily combined with the volume-stabilizing solutions for the $L_{\text {dil }}$ Lagrangian. The question arises whether the volume-stabilizing term $V(q)$ is sufficient for the existence of an open set of bounded solutions. The answer is affirmative.

Proposition 4. The general solutions for $\operatorname{SL}(n, \mathbf{R})$-restricted geodetic systems based on $T_{\mathrm{int}}^{\text {right }}, T_{\mathrm{int}}^{\text {left }}$ contain open subsets of bounded solutions. The general solutions of these systems non-restricted from $\mathrm{GL}^{+}(n, \mathbf{R})$ to $\mathrm{SL}(n, \mathbf{R})$ but modified by volume-stabilizing terms $V(q)$ also contain open subsystems of bounded solutions.

It is impossible to report the details here. Everything follows from the fact that the corresponding geodetic Hamiltonians for $T_{\text {int }}^{\text {right }}, T_{\text {int }}^{\text {left }}$ may be respectively expressed as follows: $\Im_{\text {int }}^{\text {right }}=\operatorname{Tr}\left(\Sigma^{2}\right) / 2 \alpha+(\operatorname{Tr} \Sigma)^{2} / 2 \beta-\operatorname{Tr}\left(S^{2}\right) / 4 \mu, \Im_{\text {int }}^{\text {left }}=\operatorname{Tr}\left(\hat{\Sigma}^{2}\right) / 2 \alpha+(\operatorname{Tr} \hat{\Sigma})^{2} / 2 \beta-\operatorname{Tr}\left(V^{2}\right) / 4 \mu$, where the inertial constants $\alpha=J+A, \beta=-(J+A)(J+A+n B) / B$ and $\mu=\left(J^{2}-A^{2}\right) / J$. It is seen that structurally these expressions differ from $\Im_{\text {int }}^{\mathrm{KC}}$ by the terms proportional to $\operatorname{Tr}\left(S^{2}\right), \operatorname{Tr}\left(V^{2}\right)$. These quantities are constants of motion of the geodetic problems based respectively on $\Im_{\text {int }}^{\text {right }}, \Im_{\text {int }}^{\text {left }}$. They remain constants of motion after introducing volume-stabilizing potentials $V(q)$, moreover, even after introducing any doubly-isotropic potential, i.e., one invariant under $\Phi \mapsto U \Phi W$, where $U, W$ run over $\operatorname{SO}(n, \mathbf{R})$. Besides, $\operatorname{Tr}\left(S^{2}\right), \operatorname{Tr}\left(V^{2}\right)$ have non-vanishing Poisson brackets only with those phase-space variables which, having compact geometry, cannot make the motion unbounded.

These problems become very lucid when one uses the two-polar decomposition of $\mathrm{GL}^{+}(n, \mathbf{R}): \Phi=L D R^{-1}$, where $L, R \in \mathrm{SO}(n, \mathbf{R}), D$ is diagonal and positive. One can introduce co-moving angular velocities $\lambda=L^{-1} d L / d t, \rho=R^{-1} d R / d t$, and quantities $q^{i}=\log D_{i i}$. By the way, the previously used $q$-variable is the "center of mass" of $q^{i}$ 's: $q=\left(q^{1}+\ldots+q^{n}\right) / n$. Then we introduce canonical spins $j, k$ conjugated respectively to $\lambda$, $\rho$, and canonical momenta $p_{i}$ conjugated to $q^{i}$. The previously used $p$-quantity is canonically conjugated to $q$. So, our phase space is "parameterized" by arrays $(j, k, q, p ; L, R)$. The subarrays $(j, k, q, p)$ are closed under Poisson brackets. In geodetic models described above Hamiltonians depend only on these quantities. The same concerns models with volume-stabilizing potentials $V(q)$ and even with all doubly-isotropic potentials $V\left(q^{i}\right)$. Formulating equations of motion in terms of Poisson brackets, $d F / d t=\{F, H\}$, and making use of the fact that $\operatorname{Tr}\left(S^{2}\right), \operatorname{Tr}\left(V^{2}\right)$ Poisson-commute with the $(j, k, q, p)$-variables [7], we conclude that all discussed here geodetic models have the same dynamics on the level of these coordinates. The difference appears only in the time evolution of $L, R$-variables. But due to the compactness of $\mathrm{SO}(n, \mathbf{R})$, this has nothing to do with the boundness of motion. An important fact: for the general geodetic models $T_{\text {int }}^{\text {right }}, T_{\text {int }}^{\text {left }}$ restricted to $\mathrm{SL}(n, \mathbf{R})$ even for $n=2,3$ the bounded solutions need not to be periodic in the total phase space $\operatorname{SL}(n, \mathbf{R}) \times \operatorname{SL}(n, \mathbf{R})^{\prime}$ (i.e., when the $L, R$-variables are included). It is interesting that the dynamics of our reduced variables $(j, k, q, p)$ is closely related to the theory of one-dimensional lattices. The quantities $q^{a}$ are then interpreted as lattice points [6], e.g., for the special case $J=0, B=0$, and $R_{a b}=-j_{a b}-k_{a b}, A_{a b}=j_{a b}-k_{a b}$, we obtain:

$$
H=\frac{1}{2 A} \sum_{a} p_{a}^{2}+\frac{1}{32 A} \sum_{a, b} \frac{R_{a b}^{2}}{\operatorname{sh}^{2}\left[\left(q^{a}-q^{b}\right) / 2\right]}-\frac{1}{32 A} \sum_{a, b} \frac{A_{a b}^{2}}{\operatorname{ch}^{2}\left[\left(q^{a}-q^{b}\right) / 2\right]} .
$$


On the quantum level the existence of an open subset of classical bounded solutions should manifest itself by the existence of a discrete energy spectrum. We mean here the traditional Schrödinger quantization. The metric tensor underlying the kinetic energy term gives rise to the Riemannian volume element and to the corresponding Hilbert space $\mathrm{L}^{2}$ of square-integrable functions. The kinetic energy operator is given by $-\left(\hbar^{2} / 2\right) \nabla$, where $\nabla$ denotes the corresponding Laplace-Beltrami operator. For any of the above classical models the Riemann volume is identical with the Haar measure $h$. Let us remind that it is given by $d h(\Phi)=(\operatorname{det} \Phi)^{-n} d \Phi_{11} \ldots d \Phi_{n n}$ on $\operatorname{GL}(n, \mathbf{R})$, or when considered on $\operatorname{GAf}(n, \mathbf{R})$, by $d h_{\mathrm{GAf}}(x, \Phi)=(\operatorname{det} \Phi)^{-n-1} d x^{1} \ldots d x^{n} d \Phi_{11} \ldots d \Phi_{n n}$. If we use the two-polar decomposition, $h$ may be expressed as $d h(\Phi)=\prod_{i \neq j}\left|\operatorname{sh}\left(q^{i}-q^{j}\right)\right| d \mu(L) d \mu(R) d q^{1} \ldots d q^{n}$, where $\mu$ is the Haar measure on $\mathrm{SO}(n, \mathbf{R})$.

According to the Peter-Weyl theorem, wave functions on $\mathrm{GL}^{+}(n, \mathbf{R})$ may be expanded as follows: $\Psi(\Phi)=\sum_{\alpha, \beta} \sum_{m, n=1}^{N(\alpha)} \sum_{k, l=1}^{N(\beta)} D_{m n}^{\alpha}(L) \Psi_{m n k l}^{\alpha \beta}(q) D_{k l}^{\beta}\left(R^{-1}\right)$, where the Greek indices label the set of irreducible unitary representations of $\mathrm{SO}(n, \mathbf{R}), N(\alpha)$ are representation dimensions, and $D_{m n}^{\alpha}$ are matrix elements of representations. Here $q$ denotes the system of all $q^{i}$ 's, not their "center". The stationary Schrödinger equation for $\Psi$ reduces to the family of Schrödinger equations $\hat{H}^{\alpha \beta} \Psi^{\alpha \beta}=E^{\alpha \beta} \Psi^{\alpha \beta}$ for matrix-valued wave amplitudes $\Psi^{\alpha \beta}$ depending only on the variables $q^{i}$. For any pair $(\alpha, \beta) \hat{H}^{\alpha \beta}$ is a matrix of second-order differential operators in $q$-variables, e.g., if $J=0, B=0$, we have:

$$
\hat{H}^{\alpha \beta} \Psi^{\alpha \beta}=-\frac{\hbar}{2 A} \nabla[q] \Psi^{\alpha \beta}+\frac{1}{32 A} \sum_{i, j} \frac{\left(\overleftarrow{S}_{i j}^{\beta}-\vec{S}_{i j}^{\alpha}\right)^{2} \Psi^{\alpha \beta}}{\operatorname{sh}^{2}\left[\left(q^{i}-q^{j}\right) / 2\right]}-\frac{1}{32 A} \sum_{i, j} \frac{\left(\overleftarrow{S}_{i j}^{\beta}+\vec{S}_{i j}^{\alpha}\right)^{2} \Psi^{\alpha \beta}}{\operatorname{ch}^{2}\left[\left(q^{i}-q^{j}\right) / 2\right]}
$$

where $\nabla[q]=(1 / P) \sum_{i=1}^{n}\left(\partial / \partial q^{i}\right) P\left(\partial / \partial q^{i}\right), P=\prod_{i \neq j}\left|\operatorname{sh}\left(q^{i}-q^{j}\right)\right|, \vec{S}_{i j}^{\alpha} \Psi^{\alpha \beta}=S_{i j}^{\alpha} \Psi^{\alpha \beta}$, $\overleftarrow{S}_{i j}^{\beta} \Psi^{\alpha \beta}=\Psi^{\alpha \beta} S_{i j}^{\beta}, S_{a b}^{\alpha}=(\hbar / i) X_{a b}^{\alpha}$. The matrices $X_{a b}^{\alpha}$ are basic exp-generators of the $\alpha$-th irreducible representation of $\mathrm{SO}(n, \mathbf{R})$. There exist pairs $(\alpha, \beta)$ for which the spectrum of $\hat{H}^{\alpha \beta}$ has a discrete part (bounded states without potentials in non-compact manifolds $\mathrm{SL}(n, \mathbf{R}))$. For $n=2$ this may be explicitly shown on simple examples.

\section{Acknowledgment}

This work is supported by the KBN grant No 8T07A 04720.

\section{References}

[1] Abraham R and Marsden J E, Foundations of Mechanics, Benjamin-Cummings, Reading, Massachusetts, 1978.

[2] Arnold V I, Mathematical Methods of Classical Mechanics, Springer Graduate Texts in Mathematics, 60, Springer-Verlag, New York, 1978.

[3] Dyson F J, Dynamics of a Spinning Gas Cloud, Jour. of Math. and Mech. 181 (1968).

[4] Sławianowski J J, The Mechanics of the Homogeneously-Deformable Body. Dynamical Models with High Symmetries, ZAMM 62 (1982), 229-240. 
[5] Sławianowski J J, Affinely-Rigid Body and Hamiltonian Systems on GL( $n, \mathbf{R})$, Rep. on Math. Phys. 261 (1988), 73-119.

[6] Sławianowski J J, Group-Theoretic Approach to Internal and Collective Degrees of Freedom in Mechanics and Field Theory, Technische Mechanik 221 (2002), 8-13.

[7] Sławianowski J J and Kovalchuk V, Invariant geodetic problems on the affine group and related Hamiltonian systems, Rep. on Math. Phys. 51 2/3 (2003), 371-379. 